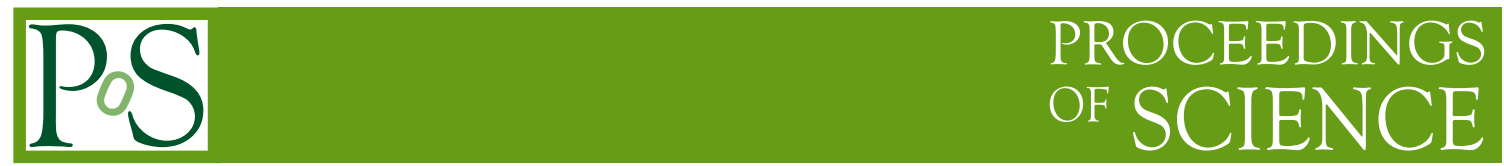

\title{
Shining Light on Dark Matter with Black Holes
}

\section{Volodymyr Takhistov*}

Kavli Institute for the Physics and Mathematics of the Universe (WPI), UTIAS

The University of Tokyo, Kashiwa, Chiba 277-8583, Japan

E-mail: volodymyr.takhistov@ipmu.jp

Primordial black holes (PBHs) from the early Universe constitute an attractive dark matter (DM) candidate. Broad classes of formation models allow for PBHs with a variety of distinct characteristics. We present several novel ideas for exploring different PBH DM parameter space regimes, including evaporating, asteroid-mass, stellar-mass as well as intermediate-mass PBHs.

The European Physical Society Conference on High Energy Physics July 26 - 30, 2021

Online conference, jointly organized by Universität Hamburg and the research center DESY

\footnotetext{
*Speaker.
} 


\section{Formation}

While astrophysical black holes are expected to form as a consequence of stellar evolution and gravitational collapse, black holes could also form in the early Universe before galaxies and stars [13]. Such primordial black holes (PBHs) can comprise a fraction or the entirety of the dark matter (DM) abundance (see [4-7] for reviews) and have been directly connected with the recent breakthrough gravitational wave (GWs) observations (e.g. [8-11]) as well as other astronomical phenomena and puzzles.

PBHs can form through a variety of mechanisms and can span decades of orders of magnitude in mass [4-7]. Typical scenarios involve collapse of an $\delta \sim O(1)$ density fluctuation that has entered the cosmological horizon after inflation. The size of the resulting PBH can then be related to the horizon mass at formation time $t$ as $M_{\mathrm{PBH}} \sim 10^{15}\left(t / 10^{-23} \mathrm{~s}\right) \mathrm{g}$. Accounting for CMB observations on larger scales, significant enhancement of power spectrum on small scales relevant for PBH formation demands fine-tuning of the inflaton potential in the simplest scenarios (e.g. [12, 13]).

PBHs with distinct features can appear in different scenarios, such as in models based on scalar fields. One such general class of models, motivated by multi-field inflation, is PBH formation from vacuum bubble "multiverse" (e.g. [14-16]). The generically complicated vacuum structure of multi-field inflation can result in vacuum bubble nucleation due to inflaton quantum-tunneling to near-by minima during slow-roll. Subsequent collapse of the expanding bubbles leads to PBHs. Continuation of such process and distribution of bubbles in size naturally leads to PBHs with an extended mass-spectrum. Intriguingly [17], this scenario can readily explain the DM abundance and is compatible with recent Subaru Hyper Suprime-Cam (HSC) microlensing observations [18]. As shown on Fig. 1, generalized models based on this setup can also simultaneously account for GW observations by the LIGO/Virgo Collaboration (LVC) as well as seeds of super-massive black holes. Exploiting PBHs, optical telescopes can also simultaneously test [19] stochastic GWs and the reported NANOGrav signal [20] in models based on flat power spectrum [21]. General classes of models based on scalar fields could also result in PBHs with non-negligible spin, such as scenarios associated with scalar fragmentation [22-24] that could be generic [25].

Spanning significant parameter space, PBHs can be associated with a multitude of distinct astronomical observations and signatures. To gain further insight into the role PBHs play in DM and physics, we discuss several novel directions for exploring PBHs over particularly intriguing mass-range regimes. This includes evaporating, asteroid-mass, stellar as well as intermediate-mass PBHs.

\section{Evaporating PBHs}

PBHs with masses $M \lesssim 10^{15} \mathrm{~g}$ undergo efficient Hawking evaporation on galactic timescales. Since PBHs will Hawking radiate covering particle spectrum of the model, evaporating PBHs can be associated with a variety of observational signatures (see [4-7]). One such possibility is heating of the surrounding interstellar medium gas by evaporating PBHs [29, 30]. Emission from evaporating PBHs thus also offers a distinct way for testing new physics beyond the Standard Model (e.g. [31, 32])

PBHs with masses $\lesssim 10^{9} \mathrm{~g}$ would have been long gone and remain unconstrained. Intriguingly, their early evaporation can imprint observable signatures. This includes contributions to dark 

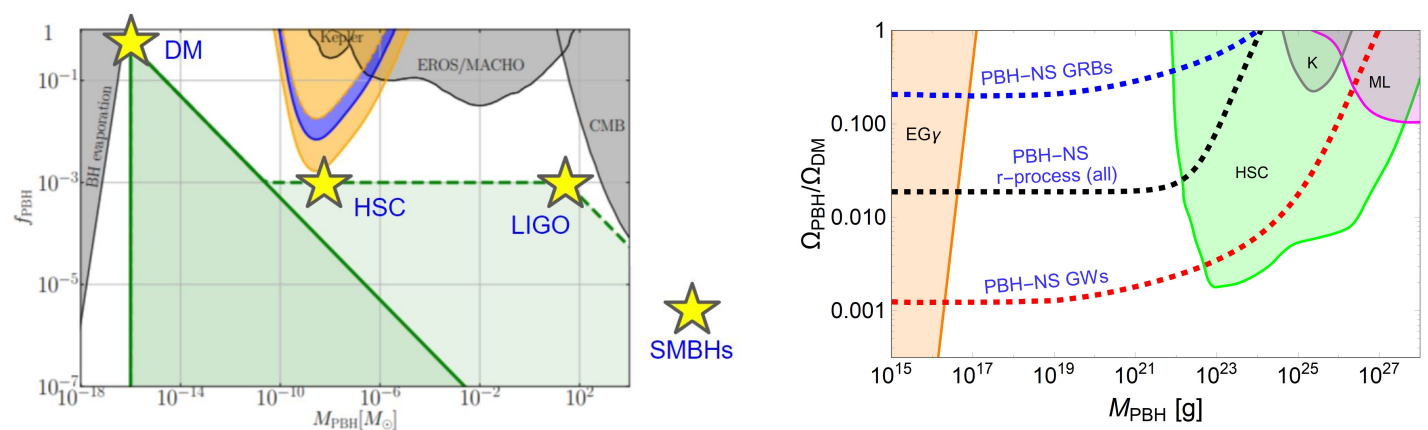

Figure 1: [Left] PBHs from a generalized model based on vacuum bubble "multiverse" scenario, consistent with observations of the DM, detected HSC and LIGO candidate events as well as seeds of supersmassive BHs, from Ref. [17]. The reach of HSC observations required to definitely test this scenario as the dominant source of PBH DM is shown in yellow. [Right] Parameter space for signatures associated with PBH DM interacting with neutron stars (denoted "PBH-NS"), including significant contributions to heavy element production in Milky Way and ultra-faint dwarf galaxies through $r$-process nucleosynthesis (black dashed) [26], "orphan gamma-ray bursts" without coincidence GWs (blue dashed) [27] as well as potentially observable GWs from merging binaries with transmuted NSs [28]. Dashed lines correspond to the maximum reach for each signal, assuming optimistic input parameters.

radiation (e.g. [33, 34]) as well as induced GWs associated with reheating due to PBH evaporation [35, 36]. As depicted on Fig. 2, coincidence signatures in GWs as well as effective number of neutrino species allow to explore PBHs over orders of magnitude in mass below $\sim 10^{9} \mathrm{~g}$ and provide insights into their spin and mass distributions as well as formation models [37].

\section{Asteroid-mass PBHs}

The parameter space window for asteroid-mass $10^{-17} M_{\odot} \lesssim M_{\mathrm{PBH}} \lesssim 10^{-12} M_{\odot}$ PBHs is unconstrained and such PBHs can constitute all of the DM abundance (e.g. [38, 39]). Compact stars, neutron stars (NS) and white dwarfs, can serve as excellent laboratories for exploring such small PBHs contributing to the DM, as shown on Fig. 1. Once captured by a star in DM-rich environment, PBH will eventually settle within and consume the host [40]. R-process nucleosynthesis associated with resulting emitted neutron-rich material establishes such systems as viable sources of heavy element production [26], the origin of which has been a long-standing mystery. Other emission signatures include [26-28] positrons and the associated $511 \mathrm{keV}$ radiation - relevant for the observed persistent Galactic Center signal (see also e.g. [41]), fast radio bursts, "orphan kilonovae" and "orphan gamma-ray bursts" without accompanying strong GWs as well as new types of microquasars. As host stars are consumed by small PBHs, a remnant population of "transmuted" solar-mass BHs will remain [28].

\section{Stellar and Intermediate-mass PBHs}

Being directly connected with GW observations (e.g. [8-11]), stellar-mass PBHs pose particular interest. Thus, identifying the origin of detected GW events is a central question with fundamental implications.

BHs with masses $\lesssim 2.5 M_{\odot}$ are not expected from conventional stellar evolution. Intriguingly, 

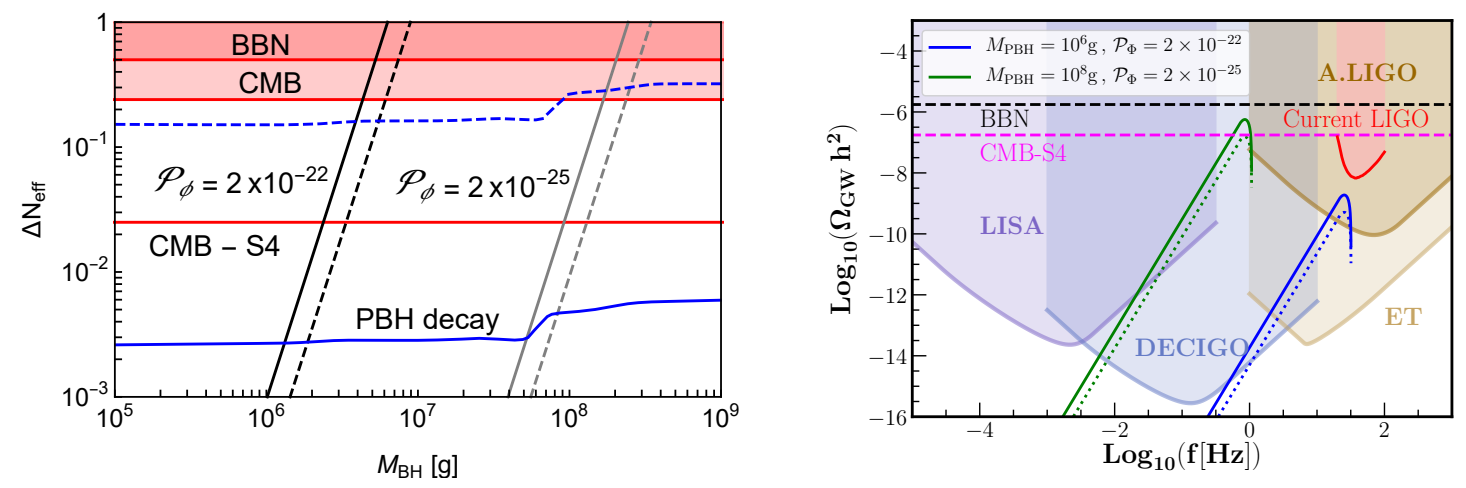

Figure 2: [Left] Contributions to effective number of neutrino species $\Delta N_{\text {eff }}$ from Hawking evaporation production ("PBH decay", blue) as well as induced GWs (with power spectrum $\mathcal{P}_{\Phi}=$ $2 \times 10^{-22}$ in black, $\mathcal{P}_{\Phi}=2 \times 10^{-25}$ in gray) for Schwarzschild ( $\operatorname{spin} a_{*}=0$, solid line) and Kerr $\left(a_{*}=0.9999\right.$, dashed), from Ref. [37]. [Right] GW spectral density induced by early isocurvature fluctuations in the scenario of reheating from PBH evaporation in terms of frequency. GWs from Kerr PBHs are shown with dashed lines.

detection of $\mathrm{a} \sim 1.5-2.6 M_{\odot} \mathrm{BH}$ candidate event GW190814C has already been reported by LVC [42]. A powerful approach to establish the origin of such BHs is to analyze their mass distribution [43]. Transmuted solar-mass BHs from (particle or PBH) DM interacting with NSs should track the known mass-distribution of NSs. As depicted on Fig. 3, events with BH masses $>1.5 M_{\odot}$ are thus unlikely to be from DM interacting with and consuming NSs.

First intermediate-mass $\mathrm{BH}$ event with a total mass of $150 M_{\odot}$ has been recently detected by LVC [44]. Residing within the pair-instability "mass-gap", this event has challenged astrophysical interpretations. While PBHs in this mass-range are subject to a variety of constraints, they often rely on a number of assumptions. A powerful cosmology-independent approach for exploring such PBHs is analyzing their interactions, energy deposition and heating of the surrounding interstellarmedium gas $[45,46]$. As shown on Fig. 3, these considerations allow to constrain PBHs over a broad mass-range of $\sim 10-10^{7} M_{\odot}$.

Aside the well studied binary BH and NS mergers, first BH-NS candidate events have been detected by LVC [47]. Similar to NS binary mergers, this class of mergers plays a central role in multi-messenger astronomy. Assuming proper binary identification, can PBHs be the source of such events? As shown by Ref. [48], the expected total merger rates of PBH-NS binaries are subdominant to the astrophysical BH-NS rates, implying that detected merger events are of astrophysical origin. While PBH-NS mergers must occur after the star formation epoch, PBH-PBH mergers can receive significant contributions from the early Universe. Thus, intriguingly, PBH-PBH mergers can account for the binary BH events observed by LVC, even with subdominant PBH-NS contributions.

\section{Summary}

PBHs make an attractive non-particle DM candidate and have been associated with a slew of distinct astrophysical phenomena. We have outlined several new ideas for exploring PBH formation as well as their detection, including evaporating, asteroid-mass, stellar as well as intermediate-mass PBHs. 

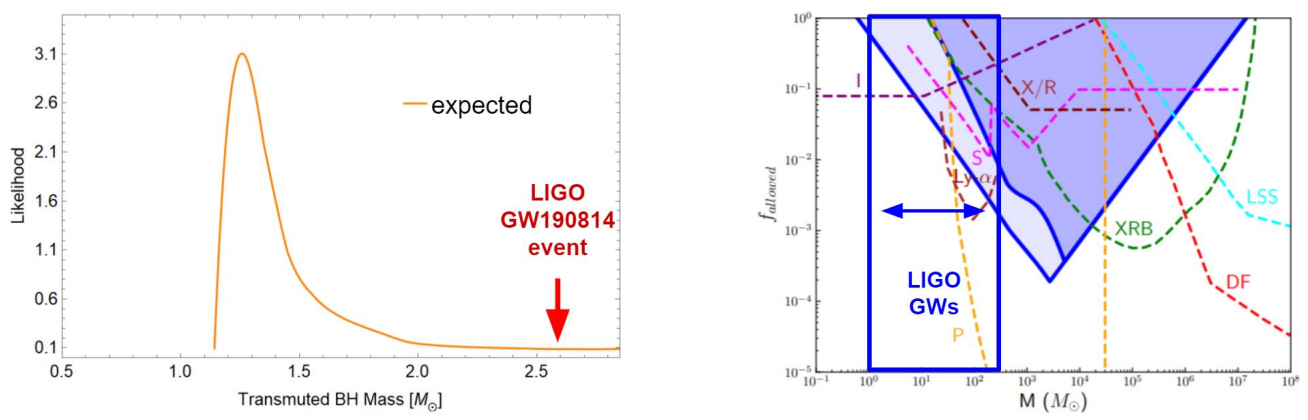

Figure 3: [Left] Expected mass distribution of "transmuted" solar-mass BHs originating from (particle or PBH) DM interacting with NSs, from [43]. [Right] Constraints on PBHs from gas heating in Leo T dwarf galaxy, from [43, 45].

Upcoming observations will allow to make definitive progress on establishing the role of PBHs in DM and physics.

\section{Acknowledgement}

The author would like to thank the organizers of the European Physical Society Conference on High Energy Physics for the opportunity to present these results. This work was supported in part by the World Premier International Research Center Initiative (WPI), MEXT, Japan.

\section{References}

[1] Y. B. Zel'dovich and I. D. Novikov, Sov. Astron. 10 (1967) 602.

[2] S. Hawking, Mon. Not. Roy. Astron. Soc. 152 (1971) 75.

[3] B. J. Carr and S. W. Hawking, Mon. Not. Roy. Astron. Soc. 168 (1974) 399.

[4] B. Carr, K. Kohri, Y. Sendouda and J. Yokoyama, 2002 .12778.

[5] M. Sasaki, T. Suyama, T. Tanaka and S. Yokoyama, Class. Quant. Grav. 35 (2018) 063001 [1801.05235].

[6] M. Yu. Khlopov, Res. Astron. Astrophys. 10 (2010) 495 [0801. 0116].

[7] A. M. Green and B. J. Kavanagh, J. Phys. G 48 (2021) 043001 [2007. 10722].

[8] T. Nakamura, M. Sasaki, T. Tanaka and K. S. Thorne, Astrophys. J. 487 (1997) L139 [astro-ph/9708060].

[9] S. Clesse and J. Garcia-Bellido, Phys. Rev. D92 (2015) 023524 [1501.07565].

[10] S. Bird et al., Phys. Rev. Lett. 116 (2016) 201301 [1603. 00464].

[11] M. Sasaki, T. Suyama, T. Tanaka and S. Yokoyama, Phys. Rev. Lett. 117 (2016) 061101 [1603.08338].

[12] M. Kawasaki and V. Takhistov, Phys. Rev. D 98 (2018) 123514 [1810. 02547].

[13] C. Germani and I. Musco, Phys. Rev. Lett. 122 (2019) 141302 [1805.04087].

[14] J. Garriga, A. Vilenkin and J. Zhang, JCAP 02 (2016) 064 [1512 . 01819].

[15] H. Deng and A. Vilenkin, JCAP 12 (2017) 044 [1710.02865].

[16] H. Deng, A. Vilenkin and M. Yamada, JCAP 07 (2018) 059 [1804 . 10059].

[17] A. Kusenko, M. Sasaki, S. Sugiyama, M. Takada, V. Takhistov and E. Vitagliano, Phys. Rev. Lett. 125 (2020) 181304 [2001.09160].

[18] H. Niikura et al., Nat. Astron. 3 (2019) 524 [1701. 02151]. 
[19] S. Sugiyama, V. Takhistov, E. Vitagliano, A. Kusenko, M. Sasaki and M. Takada, Phys. Lett. B 814 (2021) 136097 [2010.02189].

[20] NANOGRav collaboration, Z. Arzoumanian et al., 2009. 04496.

[21] V. De Luca, G. Franciolini and A. Riotto, 2009.08268.

[22] E. Cotner and A. Kusenko, Phys. Rev. Lett. 119 (2017) 031103 [1612 . 02529].

[23] E. Cotner, A. Kusenko and V. Takhistov, Phys. Rev. D 98 (2018) 083513 [1801.03321].

[24] E. Cotner, A. Kusenko, M. Sasaki and V. Takhistov, JCAP 10 (2019) 077 [1907. 10613].

[25] A. Kusenko, V. Takhistov, M. Yamada and M. Yamazaki, Phys. Lett. B 804 (2020) 135369 [1908.10930].

[26] G. M. Fuller, A. Kusenko and V. Takhistov, Phys. Rev. Lett. 119 (2017) 061101 [1704.01129].

[27] V. Takhistov, Phys. Lett. B 789 (2019) 538 [1710.09458].

[28] V. Takhistov, Phys. Lett. B 782 (2018) 77 [1707.05849].

[29] H. Kim, 2007.07739.

[30] R. Laha, P. Lu and V. Takhistov, Phys. Lett. B 820 (2021) 136459 [2009. 11837].

[31] M. J. Baker and A. Thamm, 2105.10506.

[32] M. Calzà, J. March-Russell and J. a. G. Rosa, 2110 . 13602.

[33] D. Hooper, G. Krnjaic, J. March-Russell, S. D. McDermott and R. Petrossian-Byrne, 2004.00618.

[34] A. Arbey, J. Auffinger, P. Sandick, B. Shams Es Haghi and K. Sinha, Phys. Rev. D 103 (2021) 123549 [2104.04051].

[35] G. Domènech, C. Lin and M. Sasaki, JCAP 04 (2021) 062 [2012 . 08151].

[36] K. Inomata, K. Kohri, T. Nakama and T. Terada, JCAP 10 (2019) 071 [1904 . 12878].

[37] G. Domènech, V. Takhistov and M. Sasaki, 2105.06816.

[38] A. Katz, J. Kopp, S. Sibiryakov and W. Xue, JCAP 1812 (2018) 005 [1807. 11495].

[39] P. Montero-Camacho, X. Fang, G. Vasquez, M. Silva and C. M. Hirata, 1906. 05950.

[40] F. Capela, M. Pshirkov and P. Tinyakov, Phys. Rev. D87 (2013) 123524 [1301.4984].

[41] G. M. Fuller, A. Kusenko, D. Radice and V. Takhistov, Phys. Rev. Lett. 122 (2019) 121101 [1811.00133].

[42] LiGO Scientific, Virgo collaboration, R. Abbott et al., Astrophys. J. Lett. 896 (2020) L44 [2006.12611].

[43] V. Takhistov, G. M. Fuller and A. Kusenko, Phys. Rev. Lett. 126 (2021) 071101 [2008.12780].

[44] LIGO Scientific, Virgo collaboration, R. Abbott et al., Phys. Rev. Lett. 125 (2020) 101102 [2009.01075].

[45] P. Lu, V. Takhistov, G. B. Gelmini, K. Hayashi, Y. Inoue and A. Kusenko, Astrophys. J. Lett. 908 (2021) L23 [2007. 02213].

[46] V. Takhistov, P. Lu, G. B. Gelmini, K. Hayashi, Y. Inoue and A. Kusenko, 2105.06099.

[47] LIGO Scientific, KAGRA, VIRGO collaboration, R. Abbott et al., Astrophys. J. Lett. 915 (2021) L5 [2106 . 15163].

[48] M. Sasaki, V. Takhistov, V. Vardanyan and Y.-1. Zhang, 2110.09509. 